\title{
Bethe Ansatz Solutions to Quasi Exactly Solvable Difference Equations ${ }^{\star}$
}

\author{
Ryu SASAKI ${ }^{\dagger}$, Wen-Li YANG $\ddagger$ and Yao-Zhong ZHANG $\S$ \\ $\dagger$ Yukawa Institute for Theoretical Physics, Kyoto University, Kyoto 606-8502, Japan \\ E-mail: ryu@yukawa.kyoto-u.ac.jp \\ ¥ Institute of Modern Physics, Northwest University, Xian 710069, P.R. China \\ E-mail: wlyang@nwu.edu.cn \\ $\S$ School of Mathematics and Physics, The University of Queensland, \\ Brisbane, QLD 4072, Australia \\ E-mail: yzz@maths.uq.edu.au
}

Received September 20, 2009, in final form November 10, 2009; Published online November 18, 2009 doi:10.3842/SIGMA.2009.104

\begin{abstract}
Bethe ansatz formulation is presented for several explicit examples of quasi exactly solvable difference equations of one degree of freedom which are introduced recently by one of the present authors. These equations are deformation of the well-known exactly solvable difference equations of the Meixner-Pollaczek, continuous Hahn, continuous dual Hahn, Wilson and Askey-Wilson polynomials. Up to an overall factor of the so-called pseudo ground state wavefunction, the eigenfunctions within the exactly solvable subspace are given by polynomials whose roots are solutions of the associated Bethe ansatz equations. The corresponding eigenvalues are expressed in terms of these roots.
\end{abstract}

Key words: Bethe ansatz solution; quasi-exactly solvable models

2000 Mathematics Subject Classification: 35Q40; 37N20; 39A70; 82B23

\section{Introduction}

Bethe ansatz method is one of the well-known solution methods for exactly solvable quantum systems (see, e.g. [1]) as well as for various spin models and statistical lattice models. In recent years the concept of exactly solvable quantum systems was drastically enlarged to include various examples of the so-called 'discrete' quantum mechanical systems [2, 3, 4, 5, 6, 7], in which the Schrödinger equation is a difference equation instead of differential. Known examples of exactly solvable 'discrete' quantum mechanics are deformations of exactly solvable quantum mechanics, in which the momentum operators appear in exponentiated forms instead of polynomials in ordinary quantum mechanics. Their eigenfunctions are $(q-)$ Askey scheme of hypergeometric orthogonal polynomials [8,9], which are deformations of the classical orthogonal polynomials, e.g. the Hermite, Laguerre and Jacobi polynomials. It is interesting to note that these examples are exactly solvable both in the Schrödinger and Heisenberg pictures [4, 5]. That is, for each Hamiltonian of these examples, the Heisenberg operator solution for a special coordinate called the 'sinusoidal' coordinate can be constructed, as well as the complete set of the eigenvalues and the corresponding eigenfunctions. The eigenfunctions consist of the above-mentioned $(q-)$ Askey scheme of hypergeometric orthogonal polynomials in the 'sinusoidal' coordinate. See $[6,7]$ for a comprehensive introduction of the 'discrete' quantum mechanics and the recent developments.

\footnotetext{
${ }^{\star}$ This paper is a contribution to the Proceedings of the 5-th Microconference "Analytic and Algebraic Methods V". The full collection is available at http://www.emis.de/journals/SIGMA/Prague2009.html
} 
The domain of the Hamiltonian, its hermiticity (self-adjointness), the exact Heisenberg operator solutions, the creation-annihilation operators and the dynamical symmetry algebras are explained in some detail.

In this paper we present Bethe ansatz formulations and solutions for a family of Quasi-Exactly Solvable (QES) difference equations, which were recently introduced by one of the present authors $[10,11]$. One of the main purposes of the present paper is to provide a good list of explicit Bethe-ansatz equations for the quasi-exactly solvable difference equations (2.11), (3.11), (3.15), (4.9), (4.17), and (5.5). The list will be helpful for future research in various contexts of mathematical physics. A quantum mechanical system is called quasi-exactly solvable (QES), if only a finite number $(\geq 2)$ of eigenvalues and corresponding eigenvectors can be obtained exactly $[12,13]$. Among various characterisation/identification of QES systems [12, 13, 14, 15], we promoted a simple view that a QES system is obtained by a certain deformation of an exactly solvable quantum system. As shown explicitly for ordinary quantum mechanics by SasakiTakasaki [15], the deformation procedure applies to systems of many degrees of freedom as well as for single degree of freedom systems. This is in sharp contrast to the $\operatorname{sl}(2, R)$ characterisation [13], whose applicability is limited to essentially single degree of freedom systems. Recently the deformation procedure was applied to exactly solvable 'discrete' quantum systems of one and many degrees of freedom to obtain corresponding 'discrete' QES systems $[10,11,16]$. In this paper we present Bethe ansatz solutions for these QES difference equations.

There are only a very limited number of examples of (quasi) exactly solvable difference equations which have been solved so far by the Bethe ansatz method. To our knowledge, the Bethe ansatz solutions were known only for some QES difference equations in connection with $U_{q}(s l(2))$ [17], and for exactly solvable difference equations [18, 19, 20] of the elliptic Rusijsenaars-Schneider system. Here we apply the Bethe ansatz formulation to several explicit examples of QES difference equations $[10,11]$ as deformation of exactly solvable 'discrete' quantum mechanics [2,3], which are difference analogues of the well-known quasi exactly solvable systems, the harmonic oscillator (with/without the centrifugal potential) deformed by a sextic potential and the $1 / \sin ^{2} x$ potential deformed by a $\cos 2 x$ potential. As will be shown explicitly in the main text, these Bethe ansatz equations (2.11), (3.11), (3.15), (4.9), (4.17), and (5.5) can be considered as deformations of the equations (2.16), (4.14), (5.9) determining the roots of the corresponding $(q-)$ Askey scheme of hypergeometric orthogonal polynomials [9] (the MeixnerPollaczek, continuous Hahn, continuous dual Hahn, Wilson and Askey-Wilson polynomials and their restrictions) which constitute the eigenfunctions of the undeformed exactly solvable quantum systems. The general structure of the quasi exactly solvable difference equations to be discussed in this paper and their solutions were explained in some detail in $[10,11,16]$, including the domains of the Hamiltonians, the Hilbert spaces and hermiticity and the role played by the pseudo ground state wavefunctions.

This paper is organised as follows. In Section 2, a QES discrete quantum mechanics is solved in the Bethe ansatz formalism. The Hamiltonian of the system is obtained by 'crossing' those of the Meixner-Pollaczek and the continuous Hahn polynomials as derived in [11]. Section 3 provides the Bethe ansatz formulation of the QES discrete quantum mechanical systems which are deformations of the harmonic oscillator with a sextic potential as derived in [10]. The corresponding eigenfunctions are deformations of the Meixner-Pollaczek and the continuous Hahn polynomials. Section 4 gives the Bethe ansatz solutions of the QES discrete quantum mechanical systems which are deformations of the harmonic oscillator with a centrifugal barrier and a sextic potential as derived in [10]. The corresponding eigenfunctions are deformations of the continuous dual Hahn and the Wilson polynomials. Section 5 offers a Bethe ansatz solution to a difference equation analogue of a QES system with the $1 / \sin ^{2} x$ potential deformed by a $\cos 2 x$ potential as derived in [10]. The corresponding eigenfunctions are deformations of the Askey-Wilson polynomials and their various restrictions $[8,9]$. The final section is for a summary and comments. 


\section{Difference equation of the Meixner-Pollaczek type}

In this section we will discuss Bethe ansatz solutions for the discrete quantum mechanics obtained by deforming that of the Meixner-Pollaczek polynomials in [11]. To be more precise, the corresponding discrete quantum mechanics is obtained by crossing those of the MeixnerPollaczek and the continuous Hahn polynomials, that is, with the quadratic potential function of the continuous Hahn polynomial multiplied by a constant phase factor $e^{-i \beta}$ of the MeixnerPollaczek type. It was shown in [11] that this system is quasi exactly solvable. The corresponding Hamiltonian is:

$$
\begin{aligned}
& \mathcal{H} \stackrel{\text { def }}{=} \sqrt{V(x)} e^{-i \partial_{x}} \sqrt{V(x)^{*}}+\sqrt{V(x)^{*}} e^{+i \partial_{x}} \sqrt{V(x)}-V(x)-V(x)^{*}+\alpha_{\mathcal{M}} x \\
&= A^{\dagger} A+\alpha_{\mathcal{M}} x, \quad \alpha_{\mathcal{M}} \stackrel{\text { def }}{=}-2 \mathcal{M} \sin \beta, \quad \mathcal{M} \in \mathbb{Z}_{+}, \\
& A^{\dagger} \stackrel{\text { def }}{=} \sqrt{V(x)} e^{-\frac{i}{2} \partial_{x}}-\sqrt{V(x)^{*}} e^{\frac{i}{2} \partial_{x}}, \quad A \stackrel{\text { def }}{=} e^{-\frac{i}{2} \partial_{x}} \sqrt{V(x)^{*}}-e^{\frac{i}{2} \partial_{x}} \sqrt{V(x)}, \\
& \\
& \quad V(x) \stackrel{\text { def }}{=}\left(a_{1}+i x\right)\left(a_{2}+i x\right) e^{-i \beta}, \quad a_{1}, a_{2} \in \mathbb{C}, \quad \operatorname{Re}\left(a_{1}\right)>0, \quad \operatorname{Re}\left(a_{2}\right)>0 .
\end{aligned}
$$

It should be noted that the Hamiltonian is no longer positive semi-definite but the hermiticity is preserved. Let us introduce the so-called pseudo ground state wavefunction $\phi_{0}(x)[10,11]$ :

$$
\phi_{0}(x) \stackrel{\text { def }}{=} e^{\beta x} \sqrt{\Gamma\left(a_{1}+i x\right) \Gamma\left(a_{2}+i x\right) \Gamma\left(a_{1}^{*}-i x\right) \Gamma\left(a_{2}^{*}-i x\right)},
$$

as the zero mode of the $A$ operator $(2.3), A \phi_{0}=0$. The similarity transformed Hamiltonian $\widetilde{\mathcal{H}}$ in terms of $\phi_{0}$,

$$
\begin{aligned}
\widetilde{\mathcal{H}} & \stackrel{\text { def }}{=} \phi_{0}^{-1} \circ \mathcal{H} \circ \phi_{0}=V(x)\left(e^{-i \partial_{x}}-1\right)+V(x)^{*}\left(e^{i \partial_{x}}-1\right)+\alpha_{\mathcal{M}} x \\
& =\left(a_{1}+i x\right)\left(a_{2}+i x\right) e^{-i \beta}\left(e^{-i \partial_{x}}-1\right)+\left(a_{1}^{*}-i x\right)\left(a_{2}^{*}-i x\right) e^{i \beta}\left(e^{i \partial_{x}}-1\right)-2 \mathcal{M} \sin \beta x,
\end{aligned}
$$

acts on the polynomial part of the wavefunction. In the exactly solvable Meixner-Pollaczek case with $V(x)=(a+i x) e^{-i \beta}$ and the continuous Hahn case with $V(x)=\left(a_{1}+i x\right)\left(a_{2}+i x\right)$, the eigenfunctions are of the form

$$
\phi_{0}(x) P(\eta(x))
$$

in which $P(\eta(x))$ is a polynomial in

$$
\eta(x)=x .
$$

After the deformation, it is obvious that $\widetilde{\mathcal{H}}$ maps a polynomial in $\eta(x)=x$ into another and it is easy to verify

$$
\widetilde{\mathcal{H}} x^{n}=2(-\mathcal{M}+n) \sin \beta x^{n+1}+\text { lower order terms, } \quad n \in \mathbb{Z}_{+} .
$$

This means that the system is not exactly solvable without the compensation term, but it is quasi exactly solvable, since $\widetilde{\mathcal{H}}$ has an invariant polynomial subspace of degree $\mathcal{M}$ :

$$
\begin{aligned}
& \widetilde{\mathcal{H}} \mathcal{V}_{\mathcal{M}} \subseteq \mathcal{V}_{\mathcal{M}} \\
& \mathcal{V}_{\mathcal{M}} \stackrel{\text { def }}{=} \operatorname{Span}\left[1, x, x^{2}, \ldots, x^{\mathcal{M}}\right], \quad \operatorname{dim} \mathcal{V}_{\mathcal{M}}=\mathcal{M}+1
\end{aligned}
$$

Let $\Psi(x)$ be one of the eigenfunctions of $\widetilde{\mathcal{H}}$ and $E$ be the corresponding eigenvalue:

$$
\widetilde{\mathcal{H}} \Psi(x)=E \Psi(x),
$$


namely,

$$
\begin{aligned}
& \left(a_{1}^{*}-i x\right)\left(a_{2}^{*}-i x\right) e^{i \beta}(\Psi(x+i)-\Psi(x)) \\
& \quad+\left(a_{1}+i x\right)\left(a_{2}+i x\right) e^{-i \beta}(\Psi(x-i)-\Psi(x))-2 \mathcal{M} \sin \beta x \Psi(x)=E \Psi(x) .
\end{aligned}
$$

Equations (2.5) and (2.6) imply that the eigenfunctions in the subspace $\mathcal{V}_{\mathcal{M}}$ have the following form

$$
\Psi(x)=\prod_{l=1}^{\mathcal{M}}\left(\eta(x)-\eta\left(x_{l}\right)\right)=\prod_{l=1}^{\mathcal{M}}\left(x-x_{l}\right),
$$

where $\left\{x_{l} \mid l=1, \ldots, \mathcal{M}\right\}$ are some parameters which will be specified later by the associated Bethe ansatz equations (2.11) below. Substituting the above equation into (2.7) and dividing both sides by $\Psi(x)$, we have

$$
E=-V(x)-V(x)^{*}-2 \mathcal{M} \sin \beta x+V(x) \prod_{l=1}^{\mathcal{M}} \frac{x-x_{l}-i}{x-x_{l}}+V(x)^{*} \prod_{l=1}^{\mathcal{M}} \frac{x-x_{l}+i}{x-x_{l}} .
$$

The r.h.s. of (2.9) is a meromorphic function of $x$, whereas the l.h.s. is a constant. To make them equal, we must null the residues of the r.h.s. It is easy to see that the singularities of the r.h.s. only appear at $x=x_{j}, j=1, \ldots, \mathcal{M}$ and $x=\infty$. The residues at $x=x_{j}$ vanish if the parameters $\left\{x_{j}\right\}$ satisfy the following Bethe ansatz equations

$$
\begin{aligned}
\prod_{l \neq j}^{\mathcal{M}} \frac{x_{j}-x_{l}-i}{x_{j}-x_{l}+i} & =\frac{V\left(x_{j}\right)^{*}}{V\left(x_{j}\right)} \frac{\eta\left(x_{j}+i\right)-\eta\left(x_{j}\right)}{\eta\left(x_{j}\right)-\eta\left(x_{j}-i\right)} \\
& =\frac{V\left(x_{j}\right)^{*}}{V\left(x_{j}\right)} e^{2 i \beta} \frac{\left(a_{1}^{*}-i x_{j}\right)\left(a_{2}^{*}-i x_{j}\right)}{\left(a_{1}+i x_{j}\right)\left(a_{2}+i x_{j}\right)}, \quad j=1, \ldots, \mathcal{M} .
\end{aligned}
$$

Throughout this paper we use the complex conjugate potential function $V(x)^{*}$ in the 'analytical' sense in $x$, that is, for complex $x$

$$
V(x)=\left(a_{1}+i x\right)\left(a_{2}+i x\right) e^{-i \beta}, \quad V(x)^{*}=\left(a_{1}^{*}-i x\right)\left(a_{2}^{*}-i x\right) e^{i \beta}, \quad x \in \mathbb{C} .
$$

This convention is necessary for the above two equations (2.10), (2.11) to be valid, since the Bethe roots $\left\{x_{j}\right\}$ are in general complex. One can check that the r.h.s. of (2.9) is indeed regular at $x=\infty$, i.e., the residue at $x=\infty$ vanishes. By the Liouville theorem the r.h.s. of (2.9) is a constant provided that $(2.11)$ is satisfied. One can get the value of the corresponding eigenvalue $E$ by taking the limit of $x \rightarrow \infty$ for the r.h.s. of (2.9). Here we present the result:

$$
E=\mathcal{M}(\mathcal{M}-1) \cos \beta+\mathcal{M}\left(\left(a_{1}+a_{2}\right) e^{-i \beta}+\left(a_{1}^{*}+a_{2}^{*}\right) e^{i \beta}\right)+2 \sin \beta \sum_{l=1}^{\mathcal{M}} x_{l},
$$

where $\left\{x_{l}\right\}$ satisfy the Bethe ansatz equation (2.11). The final term can be written as

$$
2 \sin \beta \sum_{l=1}^{\mathcal{M}} \eta\left(x_{l}\right)
$$

The wavefunction $\Psi(x)(2.8)$ becomes the eigenfunction of $\widetilde{\mathcal{H}}$ in the subspace $\mathcal{V}_{\mathcal{M}}(2.6)$ provided that the roots of the polynomial $\Psi(x)(2.8)$ are the solutions of (2.11), and then the corresponding eigenvalue is given by (2.12). Since all the roots $\left\{x_{l}\right\}$ are on the same footing, it is natural that the eigenvalue $E$ depends on the symmetric combination of them (2.13). 
A few corollaries ensue from these results. For the special case of $\beta=0$, the Hamiltonian (2.1) is exactly solvable and the corresponding eigenvectors are related to the continuous Hahn polynomials. In fact, we obtain from (2.12)

$$
\lim _{\beta \rightarrow 0} E=\mathcal{M}\left(\mathcal{M}+a_{1}+a_{2}+a_{1}^{*}+a_{2}^{*}-1\right),
$$

which is the eigenvalue corresponding to the degree $\mathcal{M}$ continuous Hahn polynomial $[2,3,6]$. The Bethe ansatz equation (2.11) now determines the zeros of the continuous Hahn polynomial. For $a_{1}, a_{2} \in \mathbb{R}_{+}$, and $a_{2} \rightarrow \infty$ limit, the above Hamiltonian $\mathcal{H}(2.1)$ divided by $a_{2}$ reduces to an exactly solvable one corresponding to the Meixner-Pollaczek polynomials [2, 3, 6]. Correspondingly the eigenvalue formula (2.12) gives

$$
\lim _{a_{2} \rightarrow \infty} E / a_{2}=2 \mathcal{M} \cos \beta .
$$

The latter is the eigenvalue of the degree $\mathcal{M}$ Meixner-Pollaczek polynomial [6]. Note that the parameter $\beta$ is related to the standard parameter $\phi$ of the Meixner-Pollaczek polynomials as $\beta=\frac{\pi}{2}-\phi$. The corresponding Bethe ansatz equation

$$
\prod_{l \neq j}^{\mathcal{M}} \frac{x_{j}-x_{l}-i}{x_{j}-x_{l}+i}=e^{2 i \beta} \frac{\left(a_{1}-i x_{j}\right)}{\left(a_{1}+i x_{j}\right)}, \quad j=1, \ldots, \mathcal{M},
$$

now determines the zeros of the degree $\mathcal{M}$ Meixner-Pollaczek polynomial. As shown in $\S 4$ of [2], this equation reduces to that determines the zeros of the Hermite polynomial in an appropriate limit.

\section{Difference equation analogue of harmonic oscillator deformed by sextic potential}

There are two types of difference equations which are difference analogues of the sextic potential Hamiltonian [10]. The Hamiltonian is given by

$$
\begin{aligned}
& \mathcal{H} \stackrel{\text { def }}{=} \sqrt{V(x)} e^{-i \partial_{x}} \sqrt{V(x)^{*}}+\sqrt{V(x)^{*}} e^{i \partial_{x}} \sqrt{V(x)}-\left(V(x)+V(x)^{*}\right)+\alpha_{\mathcal{M}}(x), \\
& \text { Type I : } \quad V(x) \stackrel{\text { def }}{=}(a+i x)(b+i x) V_{0}(x), \quad V_{0}(x) \stackrel{\text { def }}{=} c+i x, \quad a, b, c \in \mathbb{R}_{+}, \\
& \text {Type II : } \quad V(x) \stackrel{\text { def }}{=}(a+i x)(b+i x) V_{0}(x), \\
& \qquad V_{0}(x) \stackrel{\text { def }}{=} 2 \mathcal{M} x^{2}, \\
& \alpha_{\mathcal{M}}(x) \stackrel{\text { def }}{=} \mathcal{M}(\mathcal{M}-1+2(a+b+c+d)) x^{2} .
\end{aligned}
$$

Here as usual $V(x)^{*}$ is the 'analytical' complex conjugate of $V(x)$.

\subsection{Type I theory}

Here we will consider the difference equation of type I, while the difference equation of type II will be given in the next subsection. If $V$ is replaced by $V_{0}$ in (3.2) and the last term in (3.1), $\alpha_{\mathcal{M}}(x)$, is removed, $\mathcal{H}$ becomes the exactly solvable Hamiltonian of a difference analogue of the harmonic oscillator, or the deformed harmonic oscillator in 'discrete' quantum mechanics $[2,3]$. Its eigenfunctions consist of the Meixner-Pollaczek polynomials, with a special phase angle $\beta=0$, 
which is a deformation of the Hermite polynomials [2, 3, 21]. The quadratic polynomial factor $(a+i x)(b+i x)$ can be considered as multiplicative deformation, although the parameters $a, b$ and $c$ are on the equal footing. On the other hand one can consider it as a multiplicative deformation by a linear polynomial in $x$ :

$$
V(x)=(a+i x) V_{01}(x), \quad V_{01}(x) \stackrel{\text { def }}{=}(b+i x)(c+i x),
$$

with $V_{01}$ describing another difference version of an exactly solvable analogue of the harmonic oscillator [2,3]. Its eigenfunctions consist of the continuous Hahn polynomials.

Next let us introduce the similarity transformation in terms of the pseudo ground state wavefunction $\phi_{0}(x)$ as the zero mode of the $A$ operator (2.3), $A \phi_{0}=0$ :

$$
\begin{aligned}
& \phi_{0}(x) \stackrel{\text { def }}{=} \sqrt{\Gamma(a+i x) \Gamma(a-i x) \Gamma(b+i x) \Gamma(b-i x) \Gamma(c+i x) \Gamma(c-i x)}, \\
& \widetilde{\mathcal{H}} \stackrel{\text { def }}{=} \phi_{0}^{-1} \circ \mathcal{H} \circ \phi_{0}=V(x)\left(e^{-i \partial_{x}}-1\right)+V(x)^{*}\left(e^{i \partial_{x}}-1\right)+2 \mathcal{M} x^{2} .
\end{aligned}
$$

Since the parity is conserved, that is

$$
\left.\mathcal{H}\right|_{x \rightarrow-x}=\mathcal{H}
$$

it is easy to verify the action of the Hamiltonian $\widetilde{\mathcal{H}}(3.5)$ on monomials of $x$ :

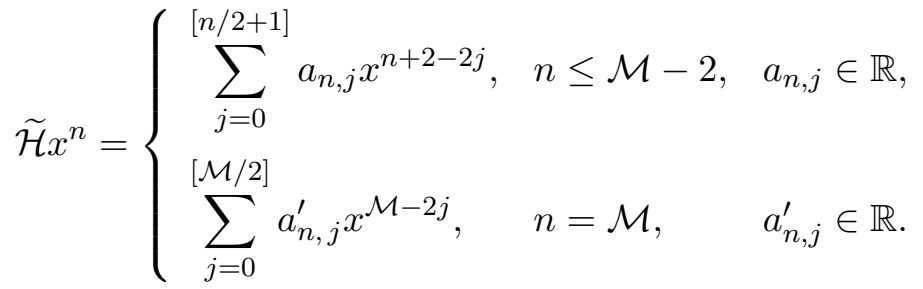

Here $[m]$ is the standard Gauss' symbol denoting the greatest integer not exceeding or equal to $m$. According to the parity of the polynomials, there are two types of invariant subspaces $\mathcal{V}_{\mathcal{M}}$ of $\widetilde{\mathcal{H}}$ :

$$
\begin{aligned}
& \widetilde{\mathcal{H}} \mathcal{V}_{\mathcal{M}} \subseteq \mathcal{V}_{\mathcal{M}}, \\
& \mathcal{V}_{\mathcal{M}} \stackrel{\text { def }}{=}\left\{\begin{array}{ll}
\operatorname{Span}\left[1, \eta(x), \ldots, \eta(x)^{k}, \ldots, \eta(x)^{\mathcal{M} / 2}\right], & \mathcal{M}: \text { even, } \\
x \operatorname{Span}\left[1, \eta(x), \ldots, \eta(x)^{k}, \ldots, \eta(x)^{(\mathcal{M}-1) / 2}\right], & \mathcal{M}: \text { odd, }
\end{array} \quad \eta(x)=x^{2},\right. \\
& \operatorname{dim} \mathcal{V}_{\mathcal{M}}= \begin{cases}\mathcal{M} / 2+1, & \mathcal{M}: \text { even, } \\
(\mathcal{M}+1) / 2, & \mathcal{M}: \text { odd. }\end{cases}
\end{aligned}
$$

\subsubsection{The case of even $\mathcal{M}$}

Let us introduce a positive integer $N$ such that $\mathcal{M}=2 N$. Equations (3.7) and (3.8) imply that the eigenfunctions of $\widetilde{\mathcal{H}}$ in the subspace $\mathcal{V}_{\mathcal{M}}$ are of the form

$$
\Psi(x)=\prod_{l=1}^{N}\left(\eta(x)-\eta\left(x_{l}\right)\right)=\prod_{l=1}^{N}\left(x-x_{l}\right)\left(x+x_{l}\right) .
$$

Analogous calculation shows that the polynomial $\Psi(x)$ becomes the eigenfunction of $\widetilde{\mathcal{H}}$ if the roots of the polynomial satisfy the Bethe ansatz equations

$$
\prod_{l \neq j}^{N} \frac{\left(x_{j}-x_{l}-i\right)\left(x_{j}+x_{l}-i\right)}{\left(x_{j}-x_{l}+i\right)\left(x_{j}+x_{l}+i\right)}=\frac{V\left(x_{j}\right)^{*}}{V\left(x_{j}\right)} \frac{\eta\left(x_{j}+i\right)-\eta\left(x_{j}\right)}{\eta\left(x_{j}\right)-\eta\left(x_{j}-i\right)}
$$




$$
=\frac{\left(a-i x_{j}\right)\left(b-i x_{j}\right)\left(c-i x_{j}\right)\left(2 x_{j}+i\right)}{\left(a+i x_{j}\right)\left(b+i x_{j}\right)\left(c+i x_{j}\right)\left(2 x_{j}-i\right)}, \quad j=1, \ldots, N .
$$

The corresponding eigenvalue $E$ is given by

$$
E=\frac{1}{3} \mathcal{M}(\mathcal{M}-1)(\mathcal{M}-2)+(a+b+c) \mathcal{M}(\mathcal{M}-1)+2(a b+a c+b c) \mathcal{M}-4 \sum_{l=1}^{N} x_{l}^{2},
$$

where $\left\{x_{l}\right\}$ satisfy the Bethe ansatz equations (3.11). Again the final term is symmetric in $\left\{x_{j}\right\}$ and can be written as $-4 \sum_{j=1}^{N} \eta\left(x_{j}\right)$.

\subsubsection{The case of odd $\mathcal{M}$}

Let us introduce a positive integer $N$ such that $\mathcal{M}=2 N+1$. (3.7) and (3.8) imply that the eigenfunctions of $\widetilde{\mathcal{H}}$ in the subspace $\mathcal{V}_{\mathcal{M}}$ are of the form

$$
\Psi(x)=x \prod_{l=1}^{N}\left(\eta(x)-\eta\left(x_{l}\right)\right)=x \prod_{l=1}^{N}\left(x-x_{l}\right)\left(x+x_{l}\right) .
$$

Analogous calculation shows that the polynomial $\Psi(x)$ becomes the eigenfunction of $\widetilde{\mathcal{H}}$ if the roots of the polynomial satisfy the Bethe ansatz equations

$$
\begin{gathered}
\frac{\left(x_{j}-i\right)}{\left(x_{j}+i\right)} \prod_{l \neq j}^{N} \frac{\left(x_{j}-x_{l}-i\right)\left(x_{j}+x_{l}-i\right)}{\left(x_{j}-x_{l}+i\right)\left(x_{j}+x_{l}+i\right)}=\frac{V\left(x_{j}\right)^{*}}{V\left(x_{j}\right)} \frac{\left(\eta\left(x_{j}+i\right)-\eta\left(x_{j}\right)\right)}{\left(\eta\left(x_{j}\right)-\eta\left(x_{j}-i\right)\right)} \\
=\frac{\left(a-i x_{j}\right)\left(b-i x_{j}\right)\left(c-i x_{j}\right)\left(2 x_{j}+i\right)}{\left(a+i x_{j}\right)\left(b+i x_{j}\right)\left(c+i x_{j}\right)\left(2 x_{j}-i\right)}, \quad j=1, \ldots, N .
\end{gathered}
$$

The corresponding eigenvalue $E$ is given by

$$
E=\frac{1}{3} \mathcal{M}(\mathcal{M}-1)(\mathcal{M}-2)+(a+b+c) \mathcal{M}(\mathcal{M}-1)+2(a b+a c+b c) \mathcal{M}-4 \sum_{l=1}^{N} x_{l}^{2},
$$

where $\left\{x_{l}\right\}$ satisfy the Bethe ansatz equations (3.15). The expression for the eigenvalue is exactly the same as the even $\mathcal{M}$ case (3.12).

In this example, exactly solvable limits are also obtained by making one or two parameters go to infinity; for example $a \rightarrow \infty$ or both $a \rightarrow \infty$ and $b \rightarrow \infty$. In the former case $(a \rightarrow \infty)$, the scaled Hamiltonian (3.1) $\mathcal{H} / a$ gives that of the continuous Hahn polynomials with real parameters $b$ and $c$. The eigenvalue formulas (3.12) and (3.16) reduce to that of the continuous Hahn polynomials $[2,3,6]$ :

$$
\lim _{a \rightarrow \infty} E / a=\mathcal{M}(\mathcal{M}+2(b+c)-1) .
$$

The Bethe ansatz equations (3.11) and (3.15) are equivalent to (2.11) with $\beta=0$ and $a_{1}=b \in$ $\mathbb{R}_{+}, a_{2}=c \in \mathbb{R}_{+}$. This assertion can be easily verified since the solutions of (2.11) are always paired $\left\{x_{j},-x_{j}\right\}$ including a zero $x_{j}=0$ for odd $\mathcal{M}$. In the latter case $(a \rightarrow \infty, b \rightarrow \infty)$, the scaled Hamiltonian $(3.1) \mathcal{H} /(a b)$ gives that of the Meixner-Pollaczek polynomials with $\beta=0$, $a=c$. The eigenvalue formulas (3.12) and (3.16) reduce to that of the Meixner-Pollaczek polynomials $[2,3,6]$ :

$$
\lim _{a, b \rightarrow \infty} E /(a b)=2 \mathcal{M} .
$$

Again the Bethe ansatz equations (3.11) and (3.15) are equivalent to (2.11) with $\beta=0$ and $a_{1}=c \in \mathbb{R}_{+}$. 


\section{$3.2 \quad$ Type II theory}

Another difference analogue of the sextic potential Hamiltonian has the same form as (3.1), with only the potential function $V(x)$ and the compensation term $\alpha_{\mathcal{M}}(x)$ are different:

$$
\begin{aligned}
& V(x) \stackrel{\text { def }}{=}(a+i x)(b+i x) V_{0}(x), \quad V_{0}(x) \stackrel{\text { def }}{=}(c+i x)(d+i x), \quad a, b, c, d \in \mathbb{R}_{+}, \\
& \alpha_{\mathcal{M}}(x) \stackrel{\text { def }}{=} \mathcal{M}(\mathcal{M}-1+2(a+b+c+d)) x^{2} .
\end{aligned}
$$

This Hamiltonian can be considered as a deformation by a quadratic polynomial factor $(a+$ $i x)(b+i x)$ of the exactly solvable 'discrete' quantum mechanics having the continuous Hahn polynomials as eigenfunctions [3], another difference analogue of the harmonic oscillator. See the comments in Section 5 of [16].

The pseudo ground state wavefunction $\phi_{0}(x)$ is

$$
\phi_{0}(x) \stackrel{\text { def }}{=} \sqrt{\Gamma(a+i x) \Gamma(a-i x) \Gamma(b+i x) \Gamma(b-i x) \Gamma(c+i x) \Gamma(c-i x) \Gamma(d+i x) \Gamma(d-i x)} .
$$

Again it has no node and it is square integrable. The similarity transformed Hamiltonian acting on the polynomial space is

$$
\begin{aligned}
\widetilde{\mathcal{H}} \stackrel{\text { def }}{=} & \phi_{0}^{-1} \circ \mathcal{H} \circ \phi_{0}=V(x)\left(e^{-i \partial_{x}}-1\right)+V(x)^{*}\left(e^{i \partial_{x}}-1\right) \\
& +\mathcal{M}(\mathcal{M}-1+2(a+b+c+d)) x^{2} .
\end{aligned}
$$

It is straightforward to verify the relationship (3.6) and to establish the existence of the invariant polynomial subspaces. In the following subsections, we present Bethe ansatz solutions to eigenfunctions and eigenvalues of the Hamiltonian (3.19).

\subsubsection{The case of even $\mathcal{M}$}

Let us introduce a positive integer $N$ such that $\mathcal{M}=2 N$ and a polynomial function $\Psi(x)$ of the form (3.9). Then $\Psi(x)$ becomes the eigenfunction of $\widetilde{\mathcal{H}}(3.19)$ with an even $\mathcal{M}: \mathcal{M}=2 N$ provided that the roots of the polynomial satisfy the Bethe ansatz equations

$$
\begin{aligned}
& \prod_{l \neq j}^{N} \frac{\left(x_{j}-x_{l}-i\right)\left(x_{j}+x_{l}-i\right)}{\left(x_{j}-x_{l}+i\right)\left(x_{j}+x_{l}+i\right)}=\frac{V\left(x_{j}\right)^{*}}{V\left(x_{j}\right)} \frac{\left(\eta\left(x_{j}+i\right)-\eta\left(x_{j}\right)\right)}{\left(\eta\left(x_{j}\right)-\eta\left(x_{j}-i\right)\right)} \\
& \quad=\frac{\left(a-i x_{j}\right)\left(b-i x_{j}\right)\left(c-i x_{j}\right)\left(d-i x_{j}\right)\left(2 x_{j}+i\right)}{\left(a+i x_{j}\right)\left(b+i x_{j}\right)\left(c+i x_{j}\right)\left(d+i x_{j}\right)\left(2 x_{j}-i\right)}, \quad j=1, \ldots, N .
\end{aligned}
$$

The corresponding eigenvalue $E$ is given by

$$
E=2 \sum_{j=1}^{4}\left(\begin{array}{c}
\mathcal{M} \\
j
\end{array}\right) \Delta_{j}-\left(4 \Delta_{3}+(4 \mathcal{M}-6)\right) \sum_{l=1}^{N} x_{l}^{2},
$$

with the binomial coefficients

$$
\left(\begin{array}{c}
\mathcal{M} \\
j
\end{array}\right)=\frac{\mathcal{M} !}{j !(\mathcal{M}-j) !}, \quad j=0, \ldots, \mathcal{M},
$$

and the coefficients $\left\{\Delta_{j} \mid j=1, \ldots, 4\right\}$, which are the elementary symmetric polynomials in the parameters $\{a, b, c, d\}$ defined by

$$
V(x)=(a+i x)(b+i x)(c+i x)(d+i x) \stackrel{\text { def }}{=} \sum_{j=0}^{4} \Delta_{j}(i x)^{j}, \quad \Delta_{4}=1 .
$$




\subsubsection{The case of odd $\mathcal{M}$}

Let us introduce a positive integer $N$ such that $\mathcal{M}=2 N+1$ and a function $\Psi(x)$ of the form (3.13). The polynomial $\Psi(x)$ becomes the eigenfunction of $\widetilde{\mathcal{H}}(3.19)$ with an odd $\mathcal{M}$ : $\mathcal{M}=2 N+1$ provided that the roots of the polynomial satisfy the Bethe ansatz equations

$$
\begin{aligned}
& \frac{\left(x_{j}-i\right)}{\left(x_{j}+i\right)} \prod_{l \neq j}^{N} \frac{\left(x_{j}-x_{l}-i\right)\left(x_{j}+x_{l}-i\right)}{\left(x_{j}-x_{l}+i\right)\left(x_{j}+x_{l}+i\right)}=\frac{V\left(x_{j}\right)^{*}}{V\left(x_{j}\right)} \frac{\left(\eta\left(x_{j}+i\right)-\eta\left(x_{j}\right)\right)}{\left(\eta\left(x_{j}\right)-\eta\left(x_{j}-i\right)\right)} \\
& \quad=\frac{\left(a-i x_{j}\right)\left(b-i x_{j}\right)\left(c-i x_{j}\right)\left(d-i x_{j}\right)\left(2 x_{j}+i\right)}{\left(a+i x_{j}\right)\left(b+i x_{j}\right)\left(c+i x_{j}\right)\left(d+i x_{j}\right)\left(2 x_{j}-i\right)}, \quad j=1, \ldots, N .
\end{aligned}
$$

The corresponding eigenvalue $E$ is given by the same expressions as (3.21)-(3.22) but with a different value of $\mathcal{M}: \mathcal{M}=2 N+1$.

\section{Difference equation analogues of harmonic oscillator with centrifugal potential deformed by sextic potential}

The Bethe ansatz solutions for the difference equation analogues of the harmonic oscillator with the centrifugal potential deformed by a sextic potential [10] are discussed here. There are two types corresponding to the linear and quadratic polynomial deformations as discussed in [10]. The corresponding exactly solvable difference equation has the Wilson polynomials $[2,3,8,9]$ as the eigenfunctions. The Hamiltonians have the same form as (3.1), (2.2) and (2.3), with only the potential function $V(x)$ and the compensation term $\alpha_{\mathcal{M}}(x)$ are different:

$$
\begin{array}{cl}
\text { Type I : } & V(x) \stackrel{\text { def }}{=}(b+i x) V_{0}(x), \quad \alpha_{\mathcal{M}}(x) \stackrel{\text { def }}{=} \mathcal{M} x^{2}, \\
\text { Type II : } & V(x) \stackrel{\text { def }}{=}(a+i x)(b+i x) V_{0}(x), \\
& \alpha_{\mathcal{M}}(x) \stackrel{\text { def }}{=} \mathcal{M}(\mathcal{M}-1+(a+b+c+d+e+f)) x^{2},
\end{array}
$$

with a common $V_{0}(x)$

$$
V_{0}(x) \stackrel{\text { def }}{=} \frac{(c+i x)(d+i x)(e+i x)(f+i x)}{2 i x(2 i x+1)}, \quad a, b, c, d, e, f \in \mathbb{R}_{+}-\{1 / 2\} .
$$

None of the parameters $a, b, c, d, e$ or $f$ should take the value $1 / 2$, since it would cancel the denominator. Because of the centrifugal barrier, the dynamics is constrained to a half line; $0<x<\infty$. The type I case can also be considered as a quadratic polynomial deformation of the exactly solvable dynamics with $V_{01}(x)$ :

$$
\text { Type I : } \quad V(x) \stackrel{\text { def }}{=}(b+i x)(c+i x) V_{01}(x), \quad V_{01}(x) \stackrel{\text { def }}{=} \frac{(d+i x)(e+i x)(f+i x)}{2 i x(2 i x+1)},
$$

which has the continuous dual Hahn polynomials $[2,3,8,9]$ as eigenfunctions. This re-interpretation does not change the dynamics, since the Hamiltonian and $A$ and $A^{\dagger}$ operators depend on $V(x)$.

\subsection{Type I theory}

Here we consider the difference equation of type I. The pseudo ground state wavefunction $\phi_{0}(x)$ is determined as the zero mode of the $A$ operator (2.3), $A \phi_{0}=0$ :

$$
\text { Type I : } \quad \phi_{0}(x) \stackrel{\text { def }}{=} \frac{\sqrt{\prod_{j=2}^{6} \Gamma\left(a_{j}+i x\right) \Gamma\left(a_{j}-i x\right)}}{\sqrt{\Gamma(2 i x) \Gamma(-2 i x)}},
$$


in which the numbering of the parameters

$$
a_{1} \stackrel{\text { def }}{=} a, \quad a_{2} \stackrel{\text { def }}{=} b, \quad a_{3} \stackrel{\text { def }}{=} c, \quad a_{4} \stackrel{\text { def }}{=} d, \quad a_{5} \stackrel{\text { def }}{=} e, \quad a_{6} \stackrel{\text { def }}{=} f,
$$

is used. It is obvious that $\phi_{0}$ has no node in the half line $0<x<\infty$.

The similarity transformed Hamiltonian acting on the polynomial space has the same form as before (2.4)

$$
\widetilde{\mathcal{H}} \stackrel{\text { def }}{=} \phi_{0}^{-1} \circ \mathcal{H} \circ \phi_{0}=V(x)\left(e^{-i \partial_{x}}-1\right)+V(x)^{*}\left(e^{i \partial_{x}}-1\right)+\mathcal{M} \eta(x) .
$$

Again the Hamiltonian is parity invariant, that is

$$
\left.\mathcal{H}\right|_{x \rightarrow-x}=\mathcal{H}
$$

Although the potential $V(x)$ has the harmful looking denominator $1 /\{2 i x(2 i x+1)\}$, it is straightforward to verify that $\widetilde{\mathcal{H}}$ maps a polynomial in $\eta(x)=x^{2}$ into another, as $2 i x+1 \propto \eta(x-i)-\eta(x)$ :

$$
\widetilde{\mathcal{H}} x^{2 n}=\left\{\begin{array}{lll}
\sum_{j=0}^{n+1} a_{n, j} x^{2 n+2-2 j}, & n \leq \mathcal{M}-1, & a_{n, j} \in \mathbb{R}, \\
\sum_{j=0}^{\mathcal{M}} a_{n, j}^{\prime} x^{2 \mathcal{M}-2 j}, & n=\mathcal{M}, & a_{n, j}^{\prime} \in \mathbb{R} .
\end{array}\right.
$$

This is because $V_{0}$, which has the above denominator, keeps the polynomial subspace of any even degree invariant, reflecting the exact solvability. In other words, the exactly solvable discrete quantum mechanics corresponding to the undeformed potentials $V_{0}(x)$ or $V_{01}(x)$ has the eigenfunction

$$
\phi_{0}(x) P(\eta(x)), \quad \eta(x)=x^{2},
$$

in which $P(\eta)$ is either the continuous dual Hahn polynomial $\left(V_{01}(x)\right.$, (4.4)), or the Wilson polynomial $\left(V_{0}(x),(4.3)\right)$. This establishes that $\widetilde{\mathcal{H}}$ keeps the polynomial space $\mathcal{V}_{\mathcal{M}}$ invariant,

$$
\begin{aligned}
& \widetilde{\mathcal{H}} \mathcal{V}_{\mathcal{M}} \subseteq \mathcal{V}_{\mathcal{M}} \\
& \mathcal{V}_{\mathcal{M}} \stackrel{\text { def }}{=} \operatorname{Span}\left[1, x^{2}, \ldots, x^{2 k}, \ldots, x^{2 \mathcal{M}}\right], \quad \operatorname{dim} \mathcal{V}_{\mathcal{M}}=\mathcal{M}+1
\end{aligned}
$$

The above equations imply that the eigenfunctions of $\widetilde{\mathcal{H}}$ in the subspace $\mathcal{V}_{\mathcal{M}}$ are of the form

$$
\Psi(x)=\prod_{l=1}^{\mathcal{M}}\left(\eta(x)-\eta\left(x_{l}\right)\right)=\prod_{l=1}^{\mathcal{M}}\left(x-x_{l}\right)\left(x+x_{l}\right) .
$$

Analogous calculation shows that the polynomial $\Psi(x)$ becomes the eigenfunction of $\widetilde{\mathcal{H}}$ if the roots of the polynomial satisfy the Bethe ansatz equations

$$
\begin{aligned}
& \prod_{l \neq j}^{\mathcal{M}} \frac{\left(x_{j}-x_{l}-i\right)\left(x_{j}+x_{l}-i\right)}{\left(x_{j}-x_{l}+i\right)\left(x_{j}+x_{l}+i\right)}=\frac{V\left(x_{j}\right)^{*}}{V\left(x_{j}\right)} \frac{\eta\left(x_{j}+i\right)-\eta\left(x_{j}\right)}{\eta\left(x_{j}\right)-\eta\left(x_{j}-i\right)} \\
& \quad=\frac{\left(b-i x_{j}\right)\left(c-i x_{j}\right)\left(d-i x_{j}\right)\left(e-i x_{j}\right)\left(f-i x_{j}\right)}{\left(b+i x_{j}\right)\left(c+i x_{j}\right)\left(d+i x_{j}\right)\left(e+i x_{j}\right)\left(f+i x_{j}\right)}, \quad j=1, \ldots, \mathcal{M} .
\end{aligned}
$$

Note that the kinematical factors $\pm 2 i x+1$ of $V(x)$ and $V(x)^{*}$ are cancelled by $\eta(x \mp i)-\eta(x)$. The corresponding eigenvalue $E$ is given by

$$
E=\frac{2}{3} \mathcal{M}(\mathcal{M}-1)(\mathcal{M}-2)+\left(b+c+d+e+f+\frac{1}{2}\right) \mathcal{M}(\mathcal{M}-1)
$$




$$
+\{b(c+d+e+f)+c(d+e+f)+d(e+f)+e f\} \mathcal{M}-\sum_{l=1}^{\mathcal{M}} x_{l}^{2},
$$

where $\left\{x_{l}\right\}$ satisfy the Bethe ansatz equations (4.9).

As expected, exactly solvable limits are obtained by two different ways; either one or two parameters go to infinity. In the former case, the scaled Hamiltonian $(3.1) \mathcal{H} / f$ gives that of the Wilson polynomials with four real parameters, $b, c, d$ and $e$. The eigenvalue formula (4.10) reduces to that of the Wilson polynomials $[2,3,6]$

$$
\lim _{f \rightarrow \infty} E / f=\mathcal{M}(\mathcal{M}+b+c+d+e-1) .
$$

In the latter case $(e, f \rightarrow \infty)$, the scaled Hamiltonian $(3.1) \mathcal{H} /(e f)$ gives that of the continuous dual Hahn polynomials with three parameters, $b, c$ and $d$. The eigenvalue formula (4.10) reduces to that of the continuous dual Hahn polynomials $[2,3,6]$

$$
\lim _{e, f \rightarrow \infty} E /(e f)=\mathcal{M}
$$

The Bethe ansatz equations (4.9) in these limits determine the zeros of the Wilson and the continuous dual Hahn polynomials, respectively:

$$
\begin{array}{ll}
f \rightarrow \infty: & \prod_{l \neq j}^{\mathcal{M}} \frac{\left(x_{j}-x_{l}-i\right)\left(x_{j}+x_{l}-i\right)}{\left(x_{j}-x_{l}+i\right)\left(x_{j}+x_{l}+i\right)}=\frac{\left(b-i x_{j}\right)\left(c-i x_{j}\right)\left(d-i x_{j}\right)\left(e-i x_{j}\right)}{\left(b+i x_{j}\right)\left(c+i x_{j}\right)\left(d+i x_{j}\right)\left(e+i x_{j}\right)}, \\
e, f \rightarrow \infty: & \prod_{l \neq j}^{\mathcal{M}} \frac{\left(x_{j}-x_{l}-i\right)\left(x_{j}+x_{l}-i\right)}{\left(x_{j}-x_{l}+i\right)\left(x_{j}+x_{l}+i\right)}=\frac{\left(b-i x_{j}\right)\left(c-i x_{j}\right)\left(d-i x_{j}\right)}{\left(b+i x_{j}\right)\left(c+i x_{j}\right)\left(d+i x_{j}\right)} .
\end{array}
$$

\subsection{Type II theory}

Here we consider the difference equation of type II. The pseudo ground state wavefunction $\phi_{0}(x)$ is determined again as the zero mode of the $A$ operator $(2.3), A \phi_{0}=0$ :

$$
\text { Type II : } \quad \phi_{0}(x) \stackrel{\text { def }}{=} \frac{\sqrt{\prod_{j=1}^{6} \Gamma\left(a_{j}+i x\right) \Gamma\left(a_{j}-i x\right)}}{\sqrt{\Gamma(2 i x) \Gamma(-2 i x)}},
$$

where the same numbering of the parameters as those in (4.5) has been used. The similarity transformed Hamiltonian acting on the polynomial space has the similar form as (4.6) but with a different potential function $V(x)$ (4.1) and compensation term $\alpha_{\mathcal{M}}(x)(4.2)$

$$
\begin{aligned}
\widetilde{\mathcal{H}} \stackrel{\text { def }}{=} \phi_{0}^{-1} \circ \mathcal{H} \circ \phi_{0}= & V(x)\left(e^{-i \partial_{x}}-1\right)+V(x)^{*}\left(e^{i \partial_{x}}-1\right) \\
& +\mathcal{M}(\mathcal{M}-1+(a+b+c+d+e+f)) x^{2} .
\end{aligned}
$$

It can be verified that the resulting Hamiltonian $\widetilde{\mathcal{H}}(4.15)$ keeps the polynomial space $\mathcal{V}_{\mathcal{M}}$ defined in (4.7) invariant. Like the type I case, this allows us to search the corresponding eigenfunction $\Psi(x)$ of form $\Psi(x)=\prod_{l=1}^{\mathcal{M}}\left(x-x_{l}\right)\left(x+x_{l}\right)$. Analogous calculation shows that the polynomial $\Psi(x)$ becomes the eigenfunction of $\widetilde{\mathcal{H}}(4.15)$ provided that the roots of the polynomial satisfy the Bethe ansatz equations

$$
\prod_{l \neq j}^{\mathcal{M}} \frac{\left(x_{j}-x_{l}-i\right)\left(x_{j}+x_{l}-i\right)}{\left(x_{j}-x_{l}+i\right)\left(x_{j}+x_{l}+i\right)}=\frac{V\left(x_{j}\right)^{*}}{V\left(x_{j}\right)} \frac{\left(\eta\left(x_{j}+i\right)-\eta\left(x_{j}\right)\right)}{\left(\eta\left(x_{j}\right)-\eta\left(x_{j}-i\right)\right)}
$$




$$
=\frac{\left(a-i x_{j}\right)\left(b-i x_{j}\right)\left(c-i x_{j}\right)\left(d-i x_{j}\right)\left(e-i x_{j}\right)\left(f-i x_{j}\right)}{\left(a+i x_{j}\right)\left(b+i x_{j}\right)\left(c+i x_{j}\right)\left(d+i x_{j}\right)\left(e+i x_{j}\right)\left(f+i x_{j}\right)}, \quad j=1, \ldots, \mathcal{M} .
$$

The corresponding eigenvalue $E$ is given by

$$
E=\Delta_{3}\left(\begin{array}{c}
\mathcal{M} \\
1
\end{array}\right)+\left(2 \Delta_{4}+\Delta_{5}\right)\left(\begin{array}{c}
\mathcal{M} \\
2
\end{array}\right)+4\left(\Delta_{5}+1\right)\left(\begin{array}{c}
\mathcal{M} \\
3
\end{array}\right)+8\left(\begin{array}{c}
\mathcal{M} \\
4
\end{array}\right)-\left(\Delta_{5}+2(\mathcal{M}-1)\right) \sum_{l=1}^{\mathcal{M}} x_{l}^{2}
$$

Here the coefficients $\left\{\Delta_{j} \mid j=1, \ldots, 6\right\}$ are the elementary symmetric polynomials in the parameters $\{a, b, c, d, e, f\}$ defined by

$$
(a+i x)(b+i x)(c+i x)(d+i x)(e+i x)(f+i x) \stackrel{\text { def }}{=} \sum_{j=0}^{6} \Delta_{j}(i x)^{j}, \quad \Delta_{6}=1 .
$$

Various limits to the exactly solvable cases are almost the same as in the type I theory and will not be listed here.

It is interesting to note that the type II Hamiltonian of Section 3, (3.1), (3.3), (3.4), is obtained from the type II Hamiltonian of Section 4, (3.1), (4.1), (4.2) as a formal limit $e \rightarrow 0$, $f \rightarrow 1 / 2$ :

$$
\left.4 \mathcal{H}_{\text {Section } 4}\right|_{e \rightarrow 0, f \rightarrow 1 / 2}=\left.\mathcal{H}_{\text {Section } 3}\right|_{\mathcal{M} \rightarrow 2 \mathcal{M}}
$$

The Bethe ansatz equations together with the eigenvalue formulas are related in similar ways.

\section{Difference equation analogue of $1 / \sin ^{2} x$ potential deformed by $\cos 2 x$ potential}

The last example is the difference analogue of the model discussed in Subsection 2.1.2 of [10], $1 / \sin ^{2} x$ potential deformed by a $\cos 2 x$ potential. In this case the corresponding exactly solvable difference equation has the Askey-Wilson polynomials $[2,3,8,9]$ as eigenfunctions. The basic idea for showing quasi exact solvability is almost the same as shown above.

As introduced and explored in [10], this system is a quasi exactly solvable deformation of the exactly solvable dynamics which has the Askey-Wilson polynomials [2, 3, 8, 9] as eigenfunctions. Here we slightly change the notation from that of [10] for consistency with the rest of this paper. The range of the parameter $x$ is now finite, to be chosen as

$$
0<x<\pi
$$

and we introduce a complex variable $z$ and the sinusoidal coordinate $\eta(x)$ :

$$
z=e^{i x}, \quad \eta(x) \stackrel{\text { def }}{=} \cos x=\left(z+z^{-1}\right) / 2 .
$$

The unit of the shift is changed from 1 to a real constant $\gamma \stackrel{\text { def }}{=} \log q, 0<q<1$. Then the shift operator $e^{\gamma p}$ can be written as

$$
e^{\gamma p}=e^{-i \gamma \frac{d}{d x}}=q^{D}, \quad D \stackrel{\text { def }}{=} z \frac{d}{d z},
$$

whose action on a function of $x$ can be expressed as $z \rightarrow q z$ :

$$
e^{\gamma p} f(x)=f(x-i \gamma)=q^{D} \check{f}(z)=q^{z \frac{d}{d z}} \check{f}(z)=\check{f}(q z), \quad \text { with } \quad f(x)=\check{f}(z) .
$$

Note that $\gamma<0$. 
The Hamiltonian takes the form

$$
\begin{aligned}
& \mathcal{H} \stackrel{\text { def }}{=} \sqrt{V(x)} e^{\gamma p} \sqrt{V(x)^{*}}+\sqrt{V(x)^{*}} e^{-\gamma p} \sqrt{V(x)}-V(x)-V(x)^{*}+\alpha_{\mathcal{M}}(x), \\
&=\sqrt{V(x)} q^{D} \sqrt{V(x)^{*}}+\sqrt{V(x)^{*}} q^{-D} \sqrt{V(x)}-\left(V(x)+V(x)^{*}\right)+\alpha_{\mathcal{M}}(x), \\
&=A^{\dagger} A+\alpha_{\mathcal{M}}(x), \quad \alpha_{\mathcal{M}}(x) \stackrel{\text { def }}{=}-2 a b c d e q^{-1}\left(1-q^{\mathcal{M}}\right) \eta(x), \\
& A^{\dagger} \stackrel{\text { def }}{=}-i\left(\sqrt{V(x)} q^{\frac{D}{2}}-\sqrt{V(x)^{*}} q^{-\frac{D}{2}}\right), \quad A \stackrel{\text { def }}{=} i\left(q^{\frac{D}{2}} \sqrt{V(x)^{*}}-q^{-\frac{D}{2}} \sqrt{V(x)}\right), \\
& V(x) \stackrel{\text { def }}{=}(1-a z) V_{0}(x), \quad V_{0}(x) \stackrel{\text { def }}{=} \frac{(1-b z)(1-c z)(1-d z)(1-e z)}{\left(1-z^{2}\right)\left(1-q z^{2}\right)}, \\
&-1<a, b, c, d, e<1 .
\end{aligned}
$$

The Hamiltonian is obtained by deforming the potential function $V_{0}(z)$ by a linear polynomial in $z$. The parameter range (5.3) could be enlarged to one real parameter (say, a) and two complex conjugate pairs (for example, $b=c^{*}, d=e^{*}$ ), but the absolute values must be less than $1,|a|<1, \ldots,|e|<1$.

The pseudo ground state wavefunction $\phi_{0}(x)$ is determined as the zero mode of the $A$ operator $(5.2), A \phi_{0}=0$ :

$$
\phi_{0}(x) \stackrel{\text { def }}{=} \sqrt{\frac{\left(z^{2}, z^{-2} ; q\right)_{\infty}}{\left(a z, a z^{-1}, b z, b z^{-1}, c z, c z^{-1}, d z, d z^{-1}, e z, e z^{-1} ; q\right)_{\infty}}},
$$

where $\left(a_{1}, \ldots, a_{m} ; q\right)_{\infty} \stackrel{\text { def }}{=} \prod_{j=1}^{m} \prod_{n=0}^{\infty}\left(1-a_{j} q^{n}\right)$. Obviously $\phi_{0}$ has no node or singularity in $0<x<\pi$. We look for exact eigenvalues and eigenfunctions of the Hamiltonian (5.1) in the form:

$$
\mathcal{H} \phi=E \phi, \quad \phi(x)=\phi_{0}(x) \Psi(\eta(x)), \quad \eta(x)=\cos x=\left(z+z^{-1}\right) / 2,
$$

in which $\Psi(\eta(x))$ is a polynomial in $\eta(x)$ or in $(z+1 / z) / 2=\cos x$. The similarity transformed Hamiltonian acting on the polynomial space has the form

$$
\widetilde{\mathcal{H}} \stackrel{\text { def }}{=} \phi_{0}^{-1} \circ \mathcal{H} \circ \phi_{0}=V(z)\left(q^{D}-1\right)+V(z)^{*}\left(q^{-D}-1\right)-a b c d e q^{-1}\left(1-q^{\mathcal{M}}\right)\left(z+\frac{1}{z}\right) .
$$

Without the deformation factor $1-a z$ and the compensation term, the above Hamiltonian $\widetilde{\mathcal{H}}$ is exactly solvable, that is, it keeps the polynomial subspace in $\eta(x)=(z+1 / z) / 2$ of any degree invariant. The deformed Hamiltonian $\widetilde{\mathcal{H}}$ is parity invariant $\left.\mathcal{H}\right|_{x \rightarrow-x}=\mathcal{H}$ and it is straightforward to show the existence of an invariant polynomial subspace:

$$
\begin{aligned}
& \widetilde{\mathcal{H}} \mathcal{V}_{\mathcal{M}} \subseteq \mathcal{V}_{\mathcal{M}} \\
& \mathcal{V}_{\mathcal{M}} \stackrel{\text { def }}{=} \operatorname{Span}\left[1, \eta(x), \ldots, \eta(x)^{k}, \ldots, \eta(x)^{\mathcal{M}}\right], \quad \operatorname{dim} \mathcal{V}_{\mathcal{M}}=\mathcal{M}+1 .
\end{aligned}
$$

The above equations imply that the eigenfunctions of $\widetilde{\mathcal{H}}$ in the subspace $\mathcal{V}_{\mathcal{M}}$ are of the form

$$
\Psi(x)=\prod_{l=1}^{\mathcal{M}}\left(\eta(x)-\eta\left(x_{l}\right)\right)=\prod_{l=1}^{\mathcal{M}}\left(\cos x-\cos x_{l}\right) \equiv P_{\mathcal{M}}(\cos x) .
$$

Analogous calculation shows that $\Psi(x)$ becomes the eigenfunction of $\widetilde{\mathcal{H}}$ if the parameters $\left\{x_{l}\right\}$ satisfy the Bethe ansatz equations

$$
\prod_{l \neq j}^{\mathcal{M}} \frac{\cos \left(x_{j}-i \gamma\right)-\cos x_{l}}{\cos \left(x_{j}+i \gamma\right)-\cos x_{l}}=\frac{V\left(x_{j}\right)^{*}}{V\left(x_{j}\right)} \frac{\eta\left(x_{j}+i \gamma\right)-\eta\left(x_{j}\right)}{\eta\left(x_{j}\right)-\eta\left(x_{j}-i \gamma\right)}
$$




$$
=\frac{\left(z_{j}-a\right)\left(z_{j}-b\right)\left(z_{j}-c\right)\left(z_{j}-d\right)\left(z_{j}-e\right)}{\left(1-a z_{j}\right)\left(1-b z_{j}\right)\left(1-c z_{j}\right)\left(1-d z_{j}\right)\left(1-e z_{j}\right) z_{j}}, \quad j=1, \ldots, \mathcal{M}
$$

Note that $z_{j}=e^{i x_{j}}$ and $\eta\left(x_{j}-i \gamma\right)=\cos \left(x_{j}-i \gamma\right)=\left(q z+q^{-1} z^{-1}\right) / 2$, etc. Again the kinematical factors $\left(1-z^{ \pm 2}\right)\left(1-q z^{ \pm 2}\right)$ of $V(x)$ and $V(x)^{*}$ are cancelled by $\eta(x \mp i \gamma)-\eta(x)$. The corresponding eigenvalue $E$ is given by

$$
\begin{aligned}
E= & (a b c d+a b c e+a b d e+a c d e+b c d e) q^{-1}\left(q^{\mathcal{M}}-1\right)+q^{-\mathcal{M}}-1 \\
& -2 a b c d e q^{\mathcal{M}-1}\left(1-q^{-1}\right) \sum_{l=1}^{\mathcal{M}} \cos x_{l},
\end{aligned}
$$

where $\left\{x_{l}\right\}$ satisfy the Bethe ansatz equations (5.5).

In this example, there are many ways to obtain exactly solvable dynamics; by making either one $(e)$, two $(d, e)$, three $(c, d, e)$, four $(b, c, d, e)$ or five $(a, b, c, d, e)$ parameters vanish. The corresponding Hamiltonians are those describing the dynamics of the Askey-Wilson, continuous dual $q$-Hahn, Al-Salam-Chihara, big $q$-Hermite and $q$-Hermite polynomials, respectively [8, 9, $2,3,6]$. The eigenvalue formula (5.6) reduces to that of the Askey-Wilson polynomials for $e=0$ $[2,3,6]$;

$$
E=\left(q^{-\mathcal{M}}-1\right)\left(1-a b c d q^{\mathcal{M}+1}\right),
$$

and to a universal formula

$$
E=q^{-\mathcal{M}}-1,
$$

for the rest $[2,3,6]$. The Bethe ansatz equations (5.5) for the restricted case of $e=0$

$$
\prod_{l \neq j}^{\mathcal{M}} \frac{\cos \left(x_{j}-i \gamma\right)-\cos x_{l}}{\cos \left(x_{j}+i \gamma\right)-\cos x_{l}}=\frac{\left(z_{j}-a\right)\left(z_{j}-b\right)\left(z_{j}-c\right)\left(z_{j}-d\right)}{\left(1-a z_{j}\right)\left(1-b z_{j}\right)\left(1-c z_{j}\right)\left(1-d z_{j}\right)}, \quad j=1, \ldots, \mathcal{M},
$$

then determine the zeros of the Askey-Wilson polynomials. Further restrictions $d, e=0, c, d, e=$ $0, b, c, d, e=0$ and $a, b, c, d, e=0$ determine the zeros of the continuous dual $q$-Hahn, Al-SalamChihara, big $q$-Hermite and $q$-Hermite polynomials, respectively $[9,6]$.

\section{Summary and comments}

We have constructed Bethe ansatz solutions for the quasi exactly solvable difference equations of one degree of freedom introduced in [10] and [11]. These quasi exactly solvable difference equations are deformations of the well-known exactly solvable difference equations of the MeixnerPollaczek, continuous Hahn, continuous dual Hahn, Wilson and Askey-Wilson polynomials. The eigenfunctions within the exactly solvable subspace are explicitly given by some polynomials (module a pseudo ground state wavefunction $\phi_{0}$ ) whose roots are solutions of the associated Bethe ansatz equations. The corresponding eigenvalues are expressed in terms of the solutions

of the Bethe ansatz equations. These are difference equation counterparts of the results of Sasaki-Takasaki [15], which gave a Bethe ansatz formulation of the QES systems corresponding to the harmonic oscillator (with/without a centrifugal barrier) deformed by a sextic potential and the $1 / \sin ^{2} x$ potential deformed by a $\cos 2 x$ potential.

As in the exactly solvable quantum mechanics, the sinusoidal coordinates $\eta(x)[4,5]$ play an essential role. The exactly solvable sector is spanned by it

$$
\operatorname{Span}\left[1, \eta(x), \ldots, \eta(x)^{k}, \ldots, \eta(x)^{\mathcal{M}}\right]
$$


then the Bethe ansatz equations take an almost universal form, (2.10), (3.10), (3.14), (3.20), (3.23), (4.8), (4.16) and (5.4). The limits or restrictions to the exactly solvable dynamics are demonstrated in detail including various eigenvalue formulas (2.14), (2.15), (3.17), (3.18), (4.11), (4.12), (5.7) and (5.8). The Bethe ansatz equations reduce to those determining the zeros of the corresponding orthogonal polynomials, for example (2.16), (4.13), (4.14) and (5.9).

All the known quasi exactly solvable dynamics have the exactly solvable subspace consisting of polynomials in a certain variable (see, e.g. [22]). Our emphasis here is that the variable is the sinusoidal coordinate which plays the central role in the corresponding exactly solvable limits $[4,5,6]$.

It should be mentioned that there exist some examples of deriving (quasi) exactly solvable difference equations in terms of Lie algebraic deformations of exactly solvable dynamics introduced by Turbiner and his collaborators [23]. These difference equations have shifts in the real direction $\psi(x \pm 1)$ and the corresponding eigenfunctions have discrete orthogonality measures, in contrast to those discussed in this paper which have pure imaginary shifts, $\psi(x \pm i)$ or $\psi(x \pm i \gamma)$, $\gamma \in \mathbb{R}$, and the corresponding eigenfunctions have continuous orthogonality measures.

The deformations of exactly solvable dynamics for obtaining the quasi exactly solvable quantum systems introduced in $[15,10,11]$ and discussed in this paper in detail, are of the simplest type, in which the compensation term is linear in the sinusoidal coordinate. Possibility of further deformations including quadratic compensation terms will be discussed in [24], in particular, for those quantum systems having discrete orthogonality measures [7].

\section{Acknowledgements}

The financial support from Australian Research Council is gratefully acknowledged. R.S. is supported in part by Grants-in-Aid for Scientific Research from the Ministry of Education, Culture, Sports, Science and Technology, No.18340061 and No.19540179. Y.Z.Z. thanks the Yukawa Institute for Theoretical Physics, Kyoto University for hospitality and financial support.

\section{References}

[1] Infeld L., Hull T.E., The factorization method, Rev. Modern Phys. 23 (1951), 21-68. Cooper F., Khare A., Sukhatme U., Supersymmetry and quantum mechanics, Phys. Rep. 251 (1995), 267385, hep-th/9405029.

[2] Odake S., Sasaki R., Shape invariant potentials in "discrete quantum mechanics", J. Nonlinear Math. Phys. 12 (2005), suppl. 1, 507-521, hep-th/0410102.

[3] Odake S., Sasaki R., Equilibrium positions, shape invariance and Askey-Wilson polynomials, J. Math. Phys. 46 (2005), 063513, 10 pages, hep-th/0410109.

Odake S., Sasaki R., Calogero-Sutherland-Moser systems, Ruijsenaars-Schneider-van Diejen systems and orthogonal polynomials, Prog. Theoret. Phys. 114 (2005), 1245-1260, hep-th/0512155.

Odake S., Sasaki R., Equilibrium positions and eigenfunctions of shape invariant ("discrete") quantum mechanics, Rokko Lectures in Mathematics (Kobe University) 18 (2005), 85-110, hep-th/0505070.

[4] Odake S., Sasaki R., Unified theory of annihilation-creation operators for solvable ("discrete") quantum mechanics, J. Math. Phys. 47 (2006), 102102, 33 pages, quant-ph/0605215.

Odake S., Sasaki R., Exact solution in the Heisenberg picture and annihilation-creation operators, Phys. Lett. B 641 (2006), 112-117, quant-ph/0605221.

[5] Odake S., Sasaki R., Exact Heisenberg operator solutions for multi-particle quantum mechanics, J. Math. Phys. 48 (2007), 082106, 12 pages, arXiv:0706.0768.

[6] Odake S., Sasaki R., Exactly solvable 'discrete' quantum mechanics; shape invariance, Heisenberg solutions, annihilation-creation operators and coherent states, Prog. Theoret. Phys. 119 (2008), 663-700, arXiv:0802.1075.

[7] Odake S., Sasaki R., Orthogonal polynomials from Hermitian matrices, J. Math. Phys. 49 (2008), 053503, 43 pages, arXiv:0712.4106. 
[8] Andrews G.E., Askey R., Roy R., Special functions, Encyclopedia of Mathematics and Its Applications, Vol. 71, Cambridge University Press, Cambridge, 1999.

[9] Koekoek R., Swarttouw R.F., The Askey-scheme of hypergeometric orthogonal polynomials and its $q$ analogue, math.CA/9602214.

[10] Sasaki R., Quasi exactly solvable difference equations, J. Math. Phys. 48 (2007), 122104, 11 pages, arXiv:0708.0702.

[11] Sasaki R., New quasi exactly solvable difference equation, J. Nonlinear Math. Phys. 15 (2008), suppl. 3, 373-384, arXiv:0712.2616.

[12] Ushveridze A.G., Quasi-exactly solvable models in quantum mechanics, Institute of Physics Publishing, Bristol, 1994.

Morozov A.Y., Perelomov A.M., Roslyi A.A., Shifman M.A., Turbiner A.V., Quasi-exactly-solvable quantal problems: one-dimensional analog of rational conformal field theories, Internat. J. Modern Phys. A 5 (1990), 803-832.

[13] Turbiner A.V., Quasi-exactly-solvable problems and sl(2) algebra, Comm. Math. Phys. 118 (1988), $467-474$.

[14] Andrianov A.A., Ioffe M.V., Spiridonov V.P., Higher-derivative supersymmetry and the Witten index, Phys. Lett. A 174 (1993), 273-279, hep-th/9303005.

Bagrov V.G., Samsonov B.F., Darboux transformation, factorization and supersymmetry in one-dimensional quantum mechanics, Theoret. and Math. Phys. 104 (1995), 1051-1060.

Klishevich S.M., Plyushchay M.S., Supersymmetry of parafermions, Modern Phys. Lett. A 14 (1999), 27392752, hep-th/9905149.

Aoyama H., Kikuchi H., Okouchi I., Sato M., Wada S., Valley views: instantons, large order behaviors, and supersymmetry, Nuclear Phys. B 553 (1999), 644-710, hep-th/9808034.

Aoyama H., Sato M., Tanaka T., General forms of a $\mathcal{N}$-fold supersymmetric family, Phys. Lett. B 503 (2001), 423-429, quant-ph/0012065.

[15] Sasaki R., Takasaki K., Quantum Inozemtsev model, quasi-exact solvability and $\mathcal{N}$-fold supersymmetry, J. Phys. A: Math. Gen. 34 (2001), 9533-9553, Corrigendum, J. Phys. A: Math. Gen. 34 (2001), 10335, hep-th/0109008.

[16] Odake S., Sasaki R., Multi-particle quasi exactly solvable difference equations, J. Math. Phys. 48 (2007), 122105, 8 pages, arXiv:0708.0716.

[17] Wiegmann P.B., Zabrodin A.V., Bethe-ansatz for Bloch electron in magnetic field, Phys. Rev. Lett. 72 (1994), 1890-1893.

Wiegmann P.B., Zabrodin A.V., Algebraization of difference eigenvalue equations related to $U_{q}\left(\mathrm{sl}_{2}\right), \mathrm{Nuclear}$ Phys. B 451 (1995), 699-724, cond-mat/9501129.

[18] Felder G., Varchenko A., Algebraic Bethe ansatz for the elliptic quantum group $E_{\tau, \eta}\left(\operatorname{sl}_{2}\right)$, Nuclear Phys. B 480 (1996), 485-503, q-alg/9605024.

[19] Hou B.Y., Sasaki R., Yang W.-L., Algebraic Bethe ansatz for the elliptic quantum group $E_{\tau, \eta}\left(\mathrm{sl}_{n}\right)$ and its applications, Nuclear Phys. B 663 (2003), 467-486, hep-th/0303077.

Hou B.Y., Sasaki R., Yang W.-L., Eigenvalues of Ruijsenaars-Schneider model associated with $A_{n-1}$ root system in Bethe ansatz formalism, J. Math. Phys. 45 (2004), 559-575, hep-th/0309194.

[20] Manojlovic N., Nagy Z., Construction of the Bethe state for the $E_{\tau, \eta}(\operatorname{so}(3))$ elliptic quantum group, $S I G M A$ 3 (2007), 004, 10 pages, math.QA/0612086.

Manojlovic N., Nagy Z., Algebraic Bethe ansatz for the elliptic quantum group $E_{\tau, \eta}\left(A_{2}^{(2)}\right)$, J. Math. Phys. 48 (2007), 123515, 11 pages, arXiv:0704.3032.

[21] Degasperis A., Ruijsenaars S.N.M., Newton-equivalent Hamiltonians for the harmonic oscillator, Ann. Physics 293 (2001), 92-109.

[22] Turbiner A.V., Quantum mechanics: problems intermediate between exactly solvable and completely unsolvable, Soviet Phys. JETP 67 (1988), 230-236.

Gonzárez-López A., Kamran N., Olver P., Normalizability of one-dimensional quasi-exactly solvable Schrödinger operators, Comm. Math. Phys. 153 (1993), 117-146.

[23] Smirnov Y., Turbiner A., Lie algebraic discretization of differential equations, Modern Phys. Lett. A 10 (1995), 1795-1802, funct-an/9501001.

Chrissomalakos C., Turbiner A., Canonical commutation relation preserving maps, J. Phys. A: Math. Gen. 34 (2001), 10475-10485, math-ph/0104004.

[24] Odake S., Sasaki R., Unified theory of exactly and quasi-exactly solvable 'discrete' quantum mechanics. I. Formalism, arXiv:0903.2604. 